CLINICAL STUDY

\title{
Growth modelling of non-functioning pituitary adenomas in patients referred for surgery
}

\author{
Juergen Honegger, Sanna Zimmermann, Tsambika Psaras, Manfred Petrick ${ }^{1}$, Michel Mittelbronn ${ }^{2}$, \\ Ulrike Ernemann ${ }^{3}$, Martin Reincke ${ }^{4}$ and Klaus Dietz ${ }^{5}$ \\ Department of Neurosurgery, University of Tuebingen, Hoppe-Seyler-Straße 3, 72076 Tuebingen, Germany, ${ }^{1}$ Department of Neurosurgery, University of \\ Freiburg, 79106 Freiburg, Germany, ${ }^{2}$ Institute of Brain Research and ${ }^{3}$ Department of Neuroradiology, University of Tuebingen, 72076 Tuebingen, \\ Germany, ${ }^{4}$ Department of Internal Medicine (Innenstadt), University of Munich, 80336 Munich, Germany and ${ }^{5}$ Department of Medical Biometry, \\ University of Tuebingen, 72070 Tuebingen, Germany
}

(Correspondence should be addressed to J Honegger; Email: juergen.honegger@med.uni-tuebingen.de)

\begin{abstract}
Objective: Recent observational studies have established progression and recurrence rates of pituitary adenomas. However, it is still unknown how individual pituitary adenomas grow over years and whether growth kinetics follow a distinct growth model. The objective of this study was to define a growth model for non-functioning pituitary adenomas.

Methods: Fifteen patients who had five or more serial high-quality examinations with magnetic resonance images or computerized tomography scans were identified among 216 patients with nonfunctioning pituitary adenomas. Tumour volumes were assessed using a stereological method based on the Cavalieri principle. Tumour growth during the observation period was analysed and different growth models were fitted to the data.

Results: Fifteen pituitary adenomas (12 recurrent tumours and 3 newly diagnosed tumours) were longitudinally observed during a median observation period of 7.4 years (range: 2.3-11.9 years). Growth kinetics could be described either by an exponential growth model (nine patients) or by a logistic model (five patients) with initial exponential growth followed by deceleration of growth. One tumour remained unchanged in size during the observation period. None of the adenomas showed accelerated growth during the observation period. Overall, the linear growth model was not suitable to describe the growth kinetics of non-functioning pituitary adenomas.

Conclusions: Our study shows that growth of pituitary adenomas can be described by distinct growth models. Knowledge of growth dynamics has implications for clinical practice and helps to adjust scanning protocols for follow-up investigations.
\end{abstract}

European Journal of Endocrinology 158 287-294

\section{Introduction}

The behaviour of most tumour entities is reflected by the respective histopathological features. It has been claimed that the pathological diagnosis vouches for the consistency of the growth behaviour of recognized tumour types (1). In benign lesions, however, histopathological features are often not useful for predicting growth velocity. Pituitary adenomas may remain quiescent over extended periods of time or grow rapidly despite their benign histological nature (2). Even modern histopathological and immunohistochemical classifications of pituitary adenomas cannot overcome this dilemma. Only a small subset of adenomas shows features of atypia or malignancy indicating increased growth potential. Despite an increasing number of observational studies of incidentally discovered asymptomatic pituitary adenomas (3-5) or post-operative residual tumours $(6,7)$, a substantial uncertainty exists concerning the natural history of pituitary adenomas (6). An increase of tumour size or quiescence between two neuroradiological studies at different points of time does not necessarily predict the future growth behaviour of a pituitary adenoma. To overcome the uncertainty regarding the growth kinetics of non-functioning pituitary adenomas, we have analysed whether growth follows a distinct growth model. We have performed a longitudinal volumetric study and have fitted different growth models to the data. Definition of adenoma growth kinetics would substantially enhance knowledge of biological behaviour, allow prediction of growth and help to determine treatment algorithms.

\section{Subjects and methods}

\section{Patient population}

Between April 1998 and February 2006, 216 patients were surgically treated by Juergen Honegger for nonfunctioning pituitary adenomas at the Departments of 
Neurosurgery in Freiburg and Tuebingen. Special attention was prospectively given to cases with several imaging studies during an observation period prior to surgery. All patients were interviewed to determine whether imaging studies had been performed at different points of time.

In 54 patients, two or more imaging studies were available. In 18 of these patients, the tumours were documented before surgery by five or more neuroimaging studies with magnetic resonance (MR) images or computerized tomography (CT) scans obtained at different points of time during an observation period. At least five consecutive data points without intervening therapy were regarded as prerequisite to appropriate analysis of the growth model.

Two cases were excluded because of insufficient resolution and quality of the imaging studies and one case because of a slice thickness exceeding $4 \mathrm{~mm}$. The remaining 15 cases were included in the present study. Of these 15 patients, 12 were observed with a residual tumour following initial operation(s) and finally presented for re-operation due to tumour progression and/or appearance of new symptoms. It was the decision of the referring centres to withhold therapy during the observation period. At the time of presentation to us, the recurrent adenomas were surgically accessible and the patients were re-operated on. None of the patients had undergone a course of radiotherapy.

The other three patients newly presented for surgical treatment after an adenoma had been observed during a certain period of time. All patients underwent endocrinological evaluation and had no clinical or biochemical signs of hormonal hypersecretion. The histopathological diagnosis was null-cell adenoma in 13 cases
(Table 1). One patient harboured a silent corticotroph adenoma (case 13). One tumour was classified as silent gonadotroph adenoma (case 15).

\section{Volumetric measurement}

All available MR images and CT scans during the observation period were retrieved for each patient. All contiguous and parallel slices through the entire tumour were scanned (ScanJet 6100/T; Hewlett-Packard, Palo Alto, CA, USA) into a personal computer at 150 or 200 dpi resolution. For the MR images, coronal sections were used for calculation of tumour volumes. For the CTscans, only the original axial sections and no reconstructions were used for volumetric measurements. The CT and the magnetic resonance imaging (MRI) sections were not thicker than $4 \mathrm{~mm}$.

In nine patients, the course of the disease was documented by MR images only; in four patients, tumour growth was documented by CT scans only; and in two patients, both methods were used.

Tumour volumes were calculated using a stereological method according to the Cavalieri principle (8). Using this method, the three-dimensional tumour volume was calculated from two-dimensional images. The tumour contour was outlined on each section (Fig. 1) and the respective two-dimensional area was calculated using an image-processing software program available in the public domain (ImageJ; National Institutes of Health, Bethesda, MD, USA). The tumour area (square centimetres) on each section was then multiplied by the thickness of the section including the intervening gaps. The resulting volumes (cubic centimetres) from all

Table 1 Summary of cases: clinical data and imaging studies.

\begin{tabular}{|c|c|c|c|c|c|c|c|c|}
\hline $\begin{array}{l}\text { Case } \\
\text { no. }\end{array}$ & $\begin{array}{l}\operatorname{Age}^{a} \\
\operatorname{sex}\end{array}$ & $\begin{array}{l}\text { Untreated/ } \\
\text { recurrent } \\
\text { adenoma }\end{array}$ & Pathology ${ }^{b}$ & $\begin{array}{c}\text { Observation } \\
\text { period } \\
\text { (years) }\end{array}$ & $\begin{array}{l}\text { Number of } \\
\text { imaging } \\
\text { data sets }\end{array}$ & $\begin{array}{l}\text { Mean } T^{\mathrm{c}} \\
\text { (years) }\end{array}$ & $\begin{array}{l}\text { Measured } \\
\text { initial tumour } \\
\text { volume }\left(\mathrm{cm}^{3}\right)\end{array}$ & $\begin{array}{c}\text { Measured } \\
\text { tumour } \\
\text { volume last } \\
\text { observation } \\
\quad\left(\mathrm{cm}^{3}\right)\end{array}$ \\
\hline 1 & $32 \mathrm{~m}$ & Recurrent & Null-cell adenoma & 8 & 6 & 1.6 & 4.6 & 20.9 \\
\hline 2 & $68 \mathrm{~m}$ & Recurrent & Null-cell adenoma & 7.3 & 8 & 1.0 & 1.1 & 6.2 \\
\hline 4 & $34 \mathrm{f}$ & No prior therapy & Null-cell adenoma & 2.8 & 5 & 0.7 & 1.6 & 2.4 \\
\hline 5 & $62 \mathrm{~m}$ & Recurrent & Null-cell adenoma & 7.7 & 9 & 1.0 & 4.2 & 9.6 \\
\hline 6 & $60 \mathrm{~m}$ & Recurrent & Null-cell adenoma & 5.2 & 6 & 1.0 & 5.2 & 5.9 \\
\hline 7 & $52 \mathrm{~m}$ & Recurrent & Null-cell adenoma & 4.8 & 9 & 0.6 & 0.7 & 3.8 \\
\hline 8 & $69 \mathrm{f}$ & Recurrent & Null-cell adenoma & 11.9 & 9 & 1.5 & 0.8 & 1.5 \\
\hline 9 & $60 \mathrm{~m}$ & Recurrent & Null-cell adenoma & 8.2 & 8 & 1.2 & 0.8 & 3.3 \\
\hline 10 & $33 \mathrm{~m}$ & Recurrent & Null-cell adenoma & 2.3 & 5 & 0.6 & 0.9 & 4.8 \\
\hline 11 & $53 \mathrm{~m}$ & No prior therapy & Null-cell adenoma & 9.8 & 5 & 2.5 & 4.2 & 12 \\
\hline 13 & $76 \mathrm{~m}$ & Recurrent & $\begin{array}{l}\text { Silent corticotroph } \\
\text { adenoma }\end{array}$ & 11.2 & 5 & 2.8 & 0.8 & 7.6 \\
\hline 14 & $73 \mathrm{f}$ & No prior therapy & Null-cell adenoma & 9.8 & 6 & 2.0 & 3.8 & 4.0 \\
\hline 15 & $56 \mathrm{~m}$ & Recurrent & $\begin{array}{l}\text { Silent gonadotroph } \\
\text { adenoma }\end{array}$ & 7.4 & 5 & 1.8 & 5.3 & 19.5 \\
\hline
\end{tabular}

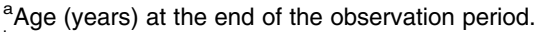

${ }^{b}$ Pathological specimen from surgery following the observation period.

${ }^{c}$ Mean $T=$ mean interval between observations. 


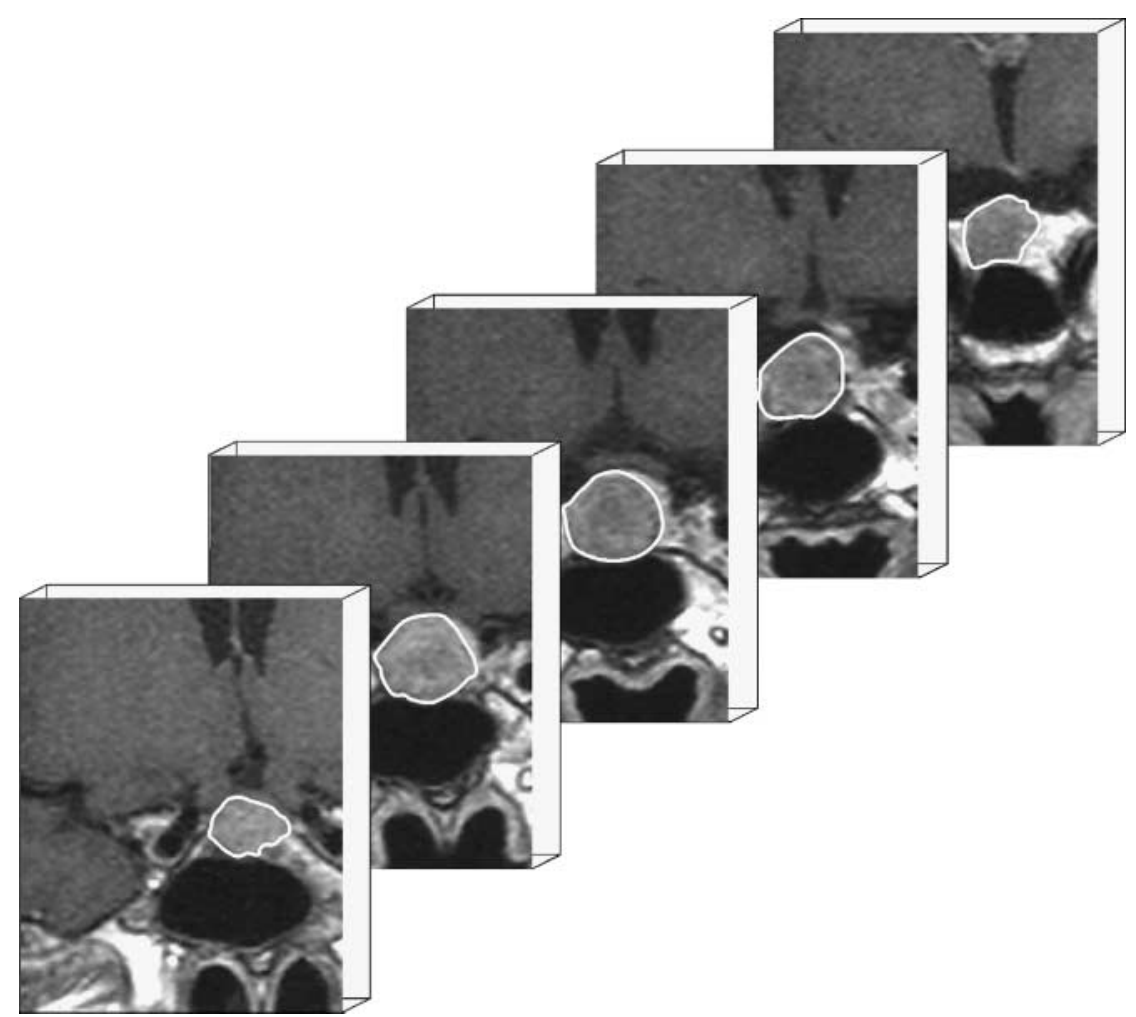

Figure 1 The magnetic resonance images (coronal section) used for volumetry show all contiguous and parallel slices through the entire tumour. The tumour contour is outlined on each slice.

contiguous sections were added and yielded the total tumour volume. The tumour contours were outlined by an experienced neuroradiologist (UE) and an experienced pituitary surgeon $(\mathrm{J} \mathrm{H})$ in joint sittings. Agreement on the tumour outlines was achieved in every case.

\section{Statistical analysis}

We assessed which mathematical model for tumour growth kinetics best describes the growth curves of nonfunctioning pituitary adenomas. All volumes are measured in $\mathrm{cm}^{3}$ and all times are measured in years.

Using the statistical software package JMP 6.0.3 (SAS Institute Inc., Carry, NC, USA), we fitted the following three established models for each patient.

Logistic The logistic model predicts initially exponential growth according to rate $\rho$, starting with volume $V_{0}$ at time $t=0$, followed by an approximately linear growth phase and finally an approach to a constant plateau $V_{1}$.

The equation for the logistic model is:

$\mathrm{V}(\mathrm{t})=\frac{\mathrm{V}_{1}}{1+\left(\frac{\mathrm{V}_{1}}{\mathrm{~V}_{0}}-1\right) \mathrm{e}^{-\rho \mathrm{t}}}$.

Exponential The exponential model predicts a stable growth rate with a constant relative (percentage) increase in tumour volume in a given period of time. Exponential growth results in a constant tumour volume doubling time (TVDT).

The equation for the exponential model is: $\mathrm{V}(\mathrm{t})=\mathrm{V}_{0} \mathrm{e}^{\rho \mathrm{t}}$.

Linear The linear model predicts a constant absolute increase in tumour volume.

The equation for the linear model is: $\mathrm{V}(\mathrm{t})=\mathrm{V}_{0}+\beta \mathrm{t}$.

The linear growth rate is $\beta$.

The parameters were estimated for each patient by a nonlinear least squares method after taking logarithms of the observed volumes and the models. The individual estimates together with their 95\% profile confidence intervals are given in Table 2 (9). The goodness of fit was assessed by calculating the coefficient of determination, i.e. the proportion of the total variance (Var) explained by the model: $r^{2}=1-$ (Var(residuals)/ Var(observations)).

The TVDT in years was calculated with the following formula: $\mathrm{TVDT}=\ln 2 / \rho$.

The logarithm of the TVDT was described by a multiple linear regression model as a function of initial age of the patients and the logarithm of the estimated initial tumour volume.

The Bartlett test was used for comparison of the measurement errors of CT and MRI volumetry. The measurement error was the percentage difference 
Table 2 Summary of the cases: growth modelling and statistical parameters.

\begin{tabular}{|c|c|c|c|c|c|}
\hline Case no. & Growth model & $\begin{array}{c}\text { Goodness of fit }=r^{2} \\
(\%)\end{array}$ & $\begin{array}{l}\text { TVDT }^{\mathrm{a}} \text { (years) } \\
\text { (95\% confidence interval) }\end{array}$ & $\begin{array}{c}\text { Estimated initial } \\
\text { tumour volume }{ }^{\mathrm{b}} V_{0} \\
\left(\mathrm{~cm}^{3}\right)(95 \% \text { confidence } \\
\text { interval })\end{array}$ & $\begin{array}{c}\text { Estimated final } \\
\text { tumour volume }{ }^{c} V_{1} \\
\left(\mathrm{~cm}^{3}\right)(95 \% \text { confidence } \\
\text { interval })\end{array}$ \\
\hline 1 & Exponential & 99.19 & $3.7(3.3-4.2)$ & $4.6(4.2-5.1)$ & - \\
\hline 2 & Logistic & 98.25 & $2.2(2.0-2.4)$ & $1.0(0.9-1.1)$ & $10.4(8.2-13.9)$ \\
\hline 3 & Exponential & 99.34 & $2.8(2.5-3.3)$ & $1.1(0.9-1.2)$ & - \\
\hline 4 & Logistic & 98.48 & $0.8(0.6-0.9)$ & $1.6(1.4-1.8)$ & $2.5(2.4-2.6)$ \\
\hline 5 & Logistic & 86.20 & $2.0(1.4-2.7)$ & $4.3(3.7-5.0)$ & $10.0(8.6-11.8)$ \\
\hline 6 & Exponential & 66.91 & $27.2(13.8-1143.4)$ & $5.0(4.6-5.4)$ & - \\
\hline 7 & Logistic & 99.31 & $1.1(0.9-1.5)$ & $0.7(0.6-0.8)$ & $5.0(3.8-8.7)$ \\
\hline 8 & Exponential & 88.69 & $12.6(9.5-18.5)$ & $0.8(0.7-0.9)$ & - \\
\hline 9 & Exponential & 90.20 & $4.3(3.2-6.4)$ & $0.8(0.6-1.0)$ & - \\
\hline 10 & Exponential & 99.95 & $1.0(0.9-1.0)$ & $0.9(0.9-0.9)$ & - \\
\hline 11 & Exponential & 98.71 & $6.4(5.3-8.1)$ & $4.2(3.7-4.6)$ & - \\
\hline 12 & Exponential & 94.61 & $10.1(8.2-13.3)$ & $3.3(3.0-3.5)$ & - \\
\hline 13 & Exponential & 95.67 & $3.1(2.2-5.1)$ & $0.6(0.3-1.2)$ & - \\
\hline 14 & Constant & - & - & & \\
\hline 15 & Logistic & 99.92 & $2.4(2.3-2.5)$ & $5.3(4.9-5.8)$ & $29.7(28.1-31.4)$ \\
\hline
\end{tabular}

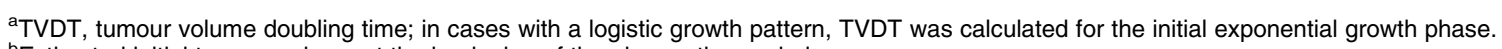

${ }^{\mathrm{b}}$ Estimated initial tumour volume at the beginning of the observation period.

${ }^{\mathrm{c}}$ Estimated final tumour volume describes the estimated final plateau in cases with a logistic growth pattern.

between the measured volume and the expected volume on the basis of the fitted growth curve.

\section{Results}

Fifteen patients with non-functioning pituitary adenomas were conservatively followed during a median observation period of 7.4 years (range: 2.3-11.9 years). The median age at the end of the observation period was 60 years (range: 32-77 years). Four patients were female and 11 were male (Table 1 ). The adenomas were followed by MR images or CT scans at five to nine different points of time (mean: 6.6 times) during the observation period (Table 1). The measured median initial tumour volume at the beginning of the observation period was $1.6 \mathrm{~cm}^{3}$ (range: $0.7-5.3 \mathrm{~cm}^{3}$ ). The median volume at the end of the observation period was $5.6 \mathrm{~cm}^{3}$ (range: $1.5-20.9 \mathrm{~cm}^{3}$ ). In preoperated cases, the mean initial and final tumour volumes were 2.4 and $7.8 \mathrm{~cm}^{3}$ respectively. Figure 2 shows an example of a longitudinal MR imaging study documenting tumour growth (case 7; Table 1).

An exponential growth curve was observed in nine cases. Figure 3 shows an example of an exponential growth curve in a 33-year-old man who presented with a recurrent non-functioning pituitary adenoma following two prior transsphenoidal operations at another centre (Table 1; case 10). The recurrent adenoma had been observed in five consecutive MR imaging studies over a period of 2.3 years. Figure $3 \mathrm{~A}$ shows the growth curve on a linear scale. The straight line on a semilogarithmic plot of tumour volume versus time (Fig. 3B) is consistent with the exponential adenoma growth and a constant TVDT. The patient harboured a rapidly growing adenoma with a TVDT of 12 months. Subsequently, the patient underwent a third transsphenoidal operation. Histological examination revealed a null-cell adenoma.

Figure 4 demonstrates a further exemplary case with exponential adenoma growth in a 67-year-old patient (Table 1; case 12). Following a transcranial operation for a non-functioning pituitary adenoma 9 years ago, the patient had an intrasellar and left parasellar residual tumour. The patient now presented with eight serial MR imaging studies documenting tumour progression during an observation period of 7.3 years. Figure $4 \mathrm{~A}$ demonstrates the growth curve of the residual adenoma on a linear scale. Tumour progression is consistent with exponential growth, as shown by a straight line on the semilogarithmic scale (Fig. 4B).

A logistic growth curve was found in five patients. In one case, tumour volume was constant throughout the observation period (Table 1; case 14).

High $r^{2}$ values were found for the exponential model (mean $r^{2}=0.93$ ) in the nine cases growing according to an exponential model. If the cases were described by the linear model, a lower $r^{2}$ of 0.88 was found. The difference was statistically significant $(P=0.015)$. In four cases with a low growth rate, however, the difference between the fitted exponential and the linear growth curves was small.

For the five cases that were best described by a logistic model, the mean $r^{2}$ value was 0.96. In all the five cases, the $r^{2}$ value was higher for the logistic model than the linear model, but the difference was not statistically significant.

Overall, the linear growth model was not suitable to describe the growth of the pituitary adenomas. The growth of pituitary adenomas was best described by the exponential model in nine cases and by the logistic model in five cases. Accelerated growth velocity was not observed during the observation period in any of the cases. 

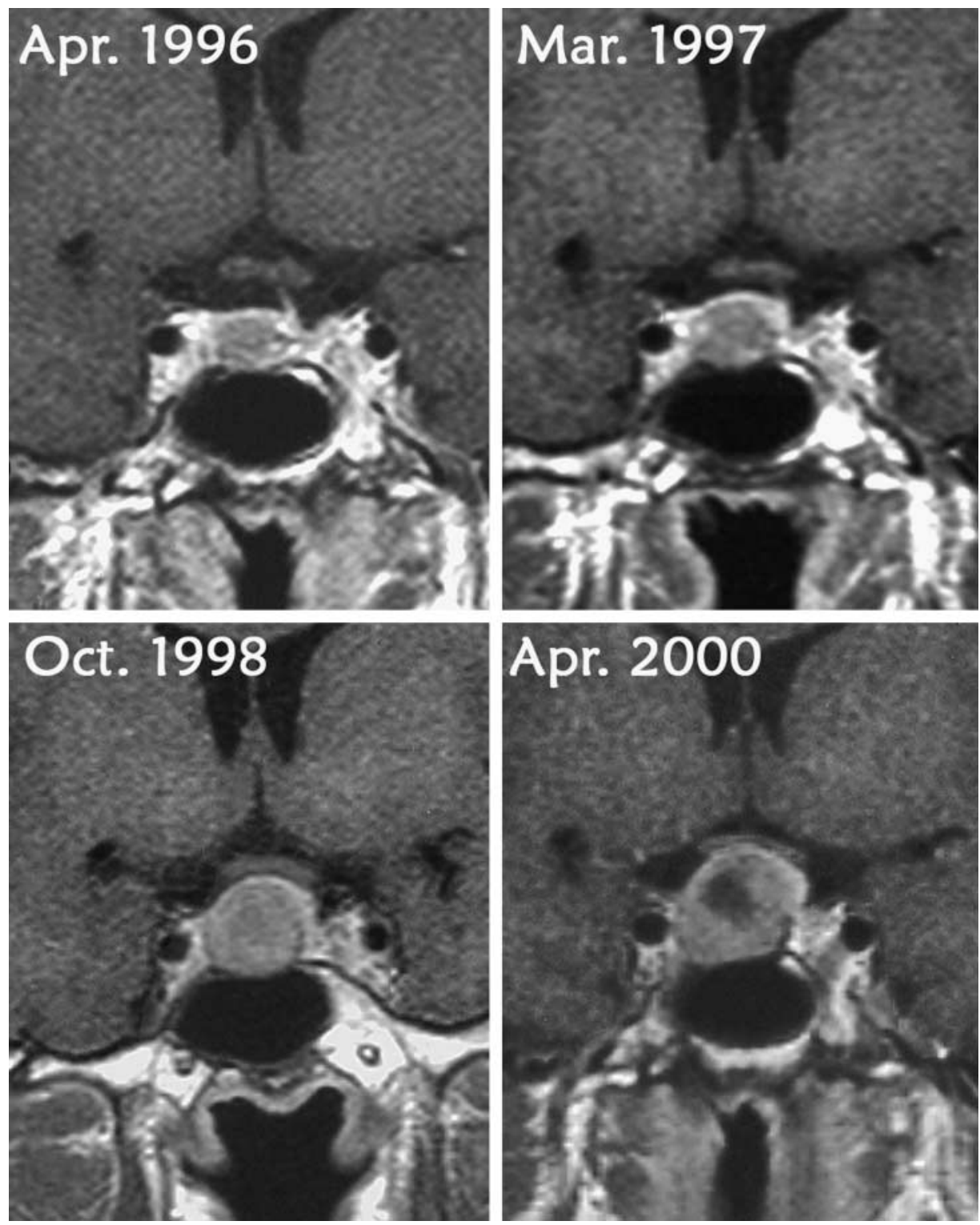

Figure 2 The magnetic resonance images demonstrate regrowth of a non-functioning pituitary adenoma during the observation period on coronal mid-tumour sections (case 7; Table 1).

The TVDTs were calculated for the 14 growing cases (Table 2). In cases with a logistic growth pattern, TVDT was calculated for the initial exponential growth phase. The median TVDT was 3.1 years (range: $0.8-27.2$ years).

A positive correlation of $r=0.44$ was found between the initial age of the patients and the logarithm of the TVDT $(P=0.12)$. Similarly, tumours with greater initial volume had a tendency towards a longer TVDT $(r=0.36$; $P=0.20$ ). When TVDT was described as a function of both a patients initial age and initial tumour volumes, the association with age was more pronounced $(P=0.07)$.

The measurement errors of CT volumetry were 2.5-fold greater than those of MRI volumetry. The difference in S.D. was highly significant $(P<0.0001)$.

\section{Discussion}

We present the first longitudinal volumetric study providing statistical evidence that non-functioning pituitary adenomas initially show an exponential growth pattern that may be followed by a deceleration of growth velocity.

Different approaches have previously been used to elucidate the prevalence and natural history of pituitary adenomas. Ezzat et al. (10) conducted a meta-analysis of all existing English language studies and found an overall prevalence of pituitary adenomas that was as high as $14.4 \%$ in selected relevant autopsy studies and $22.5 \%$ in radiological studies. The study provides knowledge on the high frequency of undiagnosed or 

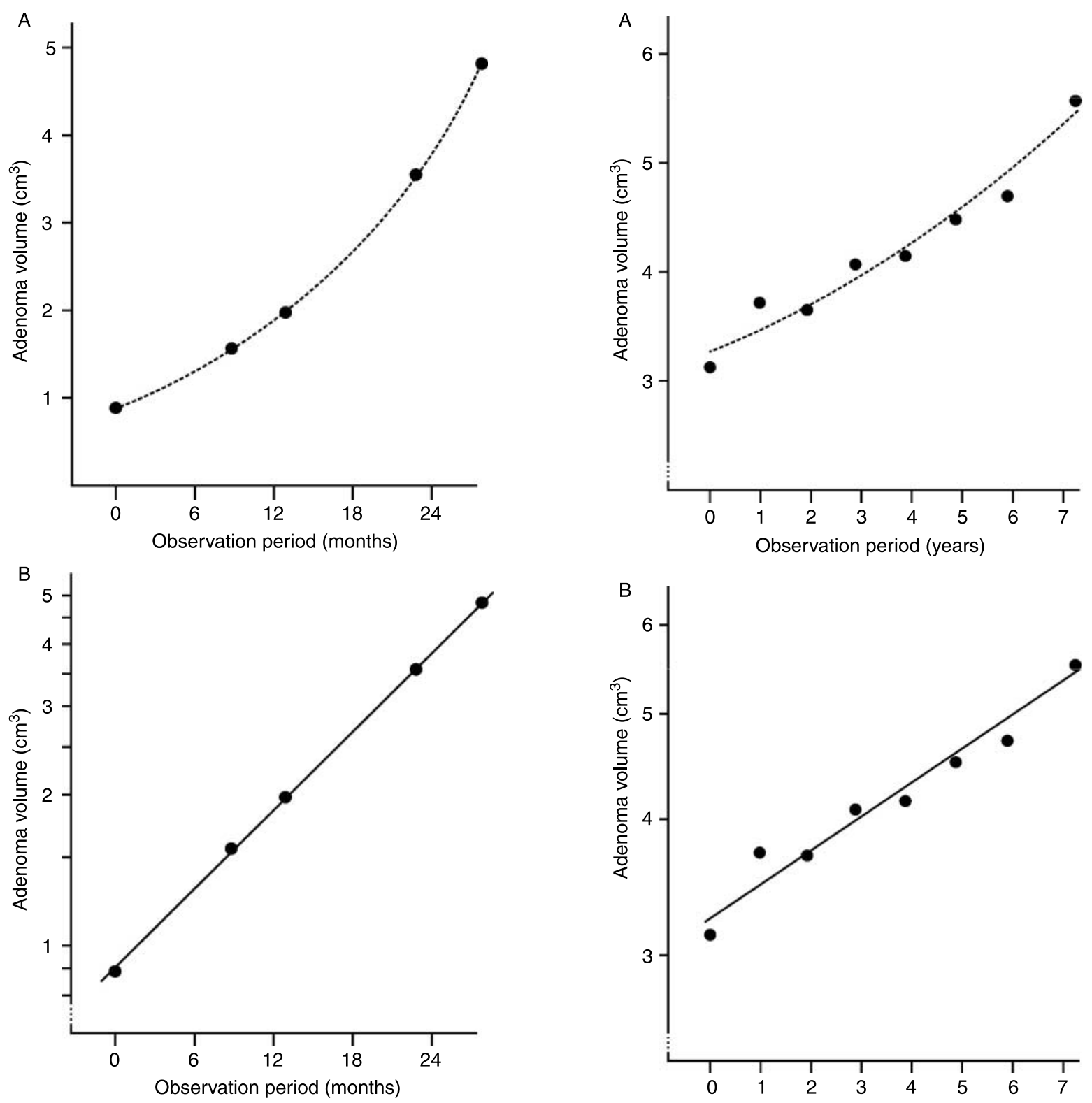

Figure 3 Longitudinal volumetric study of tumour regrowth in a 33-year-old patient following two prior operations (case 10; Table 1). Regrowth is shown on both (A) a linear plot and (B) a semilogarithmic plot of tumour volume versus time. The adenoma shows a strictly exponential growth pattern.

incidentally discovered pituitary adenomas (so-called incidentalomas). Daly et al. (11) performed a crosssectional study in the province of Liège (Belgium) and found 94 cases per 100000 population with clinically relevant pituitary adenomas. Non-functioning pituitary adenomas comprised $14.7 \%$ of these cases.

Several observational studies have emerged providing knowledge about the rate of incidentalomas that grow during the follow-up period. However, heterogeneous criteria were used for defining tumour growth. Reincke et al. (4) described tumour growth in 3 of 14

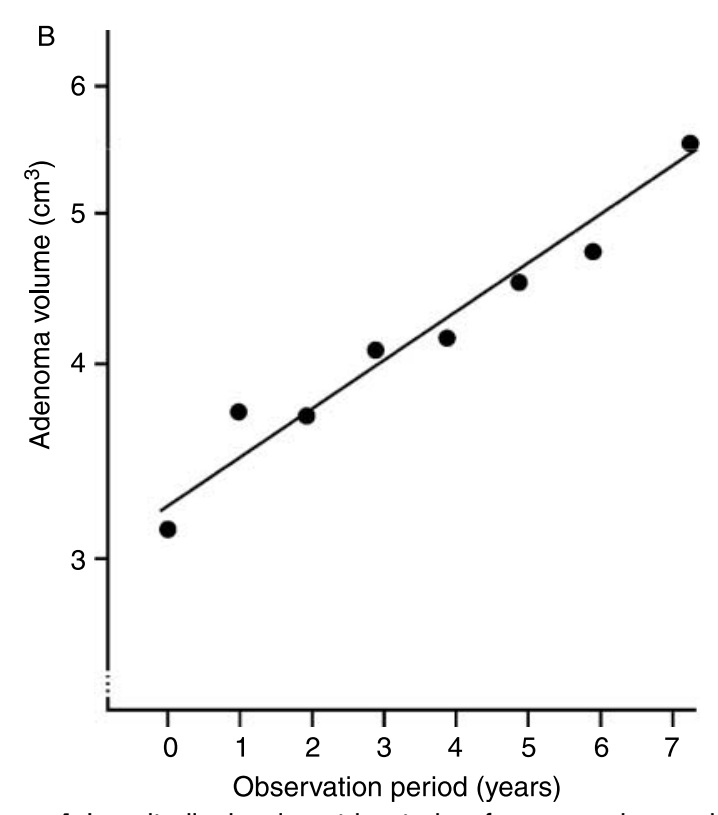

Figure 4 Longitudinal volumetric study of a regrowing residual adenoma in a 67-year-old man (case 12; Table 1). Regrowth is shown on both (A) a linear plot and (B) a semilogarithmic plot of tumour volume versus time. The straight line of progression on the semilogarithmic scale is consistent with the exponential adenoma growth.

incidentalomas at a mean follow-up of only 22 months. The majority of the tumours were in the macroadenoma stage at the time of initial detection. Dekkers et al. (12) evaluated the natural history of 28 non-functioning pituitary macroadenomas with a mean follow-up period of 85 months. Radiological growth was observed in 14 cases $(50 \%)$. In a Japanese survey (13), 20\% of non-operated suspected non-functioning adenomas developed an enlargement during a mean follow-up 
period of 50.7 months. Donovan \& Corenblum (5) found an increase in 4 of 16 pituitary incidentalomas with an initial size exceeding $10 \mathrm{~mm}$ but no increase in 15 incidentalomas smaller than $10 \mathrm{~mm}$ in size during a follow-up period of 6.1 and 6.7 years respectively. One can conclude that only a minority of pituitary adenomas exhibit considerable growth and become clinically relevant, while the majority remain stable or show extremely slow growth velocity. Tumour enlargement is more likely in macroadenomas than in microadenomas.

Other publications focus on the regrowth rate of residual non-functioning adenomas following surgical therapy $(6,7)$. Turner et al. (6) observed tumour regrowth or new symptoms in 21 of 65 patients $(32 \%)$ following transsphenoidal surgery for non-functioning adenomas at a mean follow-up of 76 months.

None of the above-mentioned studies provides quantitative information on growth velocity or tumour growth dynamics of pituitary adenomas.

Mathematical modelling has been used to describe tumour growth dynamics in other tumour entities. The best-fitting mathematical model has been analysed for different experimental in vitro and in vivo settings (14-17). In various experimental tumour paradigms, growth follows a sigmoid curve with three distinct phases: the initial exponential phase, the linear phase and the plateau due to final slowing of growth $(16,18)$. Such complex growth models with different growth phases are assumed if tumour growth is observed over a wide range of sizes from genesis to final extension (16). The logistic mathematical model is suitable to describe initial exponential growth, followed by an approximately linear growth phase and finally a constant plateau.

Final deceleration of growth has been related to impaired blood supply and central necrosis with active growth restricted to the periphery of the tumour. It is expected in rapidly growing and malignant tumours (14). If the entire tissue volume of a solid tumour was growing and the growth rate constant, it would result in exponential tumour growth $(1,14)$. In exponentially growing tumours, growth velocity can be described by the TVDT (1).

First efforts to describe human tumour growth were based on the conventional X-ray studies $(1,19)$. The advent of CT and later MR imaging was a precondition for the volumetric assessment of tumour growth in clinical settings (20, 21).

Based on imaging studies, some groups have assessed TVDTs in meningiomas $(20,22)$. They argued that volumetry and assessment of TVDT is especially suitable for meningiomas because meningiomas are well demarcated and homogeneous on contrastenhanced scans. Similarly, pituitary adenomas are generally homogeneous and solid, and tumour necrosis or cystic tumours are less common. Given the solid nature of pituitary adenomas, stable biological behaviour would result in exponential tumour growth and a constant TVDT.

So far, the TVDT of pituitary adenomas has only been assessed by a couple of groups. Ekramullah et al. (23) found that the doubling times of pituitary adenomas ranged from 200 to 2550 days. They calculated TVDT by comparing the initial and final tumour sizes. Tanaka et al. (24) followed post-operative residual tumours with serial MR images and found growth in 38 of 40 residual tumours. TVDT ranged from 506 to 5378 days (mean: 1836 days). These studies using TVDT in pituitary adenomas are based on the assumption of exponential growth kinetics. The TVDT in the exponential growth phase of our growing adenomas ranged from 0.8 to 27.2 years. In cases with logistic growth, TVDT will become longer during the further course of observation.

Our volumetric study provides direct statistical evidence that non-functioning pituitary adenomas show an exponential growth pattern. Our initial impression that pituitary adenomas exhibit linear growth (25) was not confirmed when sophisticated statistical methods were applied. The tumour volumes in our study were between 0.7 and $20.9 \mathrm{~cm}^{3}$. This range might be representative for adenomas that are conservatively followed over a certain period of time. Of course, from our results, we cannot predict whether and when the adenomas might ultimately follow a logistic growth pattern with more advanced disease. Five of the 15 cases with initial exponential growth showed decelerated growth during the observation period consistent with a logistic growth pattern.

Our cases were collected from a neurosurgical series of non-functioning adenomas. Of the 15 patients, 12 had surgery with partial tumour removal prior to the observation period. Hence, one has to take into consideration that growth patterns might differ in series of non-operated patients.

The finding in our study that non-functioning pituitary adenomas grow with a stable TVDT has important clinical implications. It has previously been claimed that the optimal scanning protocol in nonfunctioning adenomas is unknown (6). Knowledge of tumour growth pattern overcomes this uncertainty. Once the growth velocity of newly diagnosed adenomas or post-surgical residual adenomas has been established, further growth velocity can be predicted. In incidentally discovered and asymptomatic non-functioning pituitary adenomas without compression of the optic pathways, we recommend control imaging after 1 year. If tumour size remains unchanged, we suggest that further control imaging will suffice at intervals of 3 or more years. Patients can be reassured that a quiescent or slowly growing adenoma is not expected to develop a more aggressive biological behaviour during the time course or to recur quickly.

In conclusion, we have shown that growth of nonfunctioning pituitary adenomas is best described with an exponential growth model. Understanding of 
biological behaviour is important to optimize patient management, develop treatment algorithms and establish follow-up protocols. Knowledge of the growth kinetics of pituitary adenomas helps to reduce health care expenses for follow-up examinations.

\section{References}

1 Collins VP, Loeffler RK \& Tivey H. Observation on growth rates of human tumors. American Journal of Roentgenology $1956 \mathbf{7 6}$ 988-1000.

2 Scheithauer BW, Gaffey TA, Lloyd RV, Sebo TJ, Kovacs KT, Horvath E, Yapicier Ö, Young WF, Meyer FB, Kuroki T, Riehle DL \& Laws ER. Pathobiology of pituitary adenomas and carcinomas. Neurosurgery 200659 341-353.

3 Arita K, Tominaga A, Sugiyama K, Eguchi K, Iida K, Sumida M, Migita K \& Kurisu K. Natural course of incidentally found nonfunctioning pituitary adenoma, with special reference to pituitary apoplexy during follow-up examination. Journal of Neurosurgery $2006 \mathbf{1 0 4} 884-891$.

4 Reincke M, Allolio B, Saeger W, Menzel J \& Winkelmann W. The 'incidentaloma' of the pituitary gland. Is neurosurgery required? Journal of the American Medical Association $19902632772-2776$.

5 Donovan LE \& Corenblum B. The natural history of the pituitary incidentaloma. Archives of Internal Medicine 1995155 181-183.

6 Turner HE, Stratton IM, Byrne JV, Adams CBT \& Wass JAH. Audit of selected patients with nonfunctioning pituitary adenomas treated without irradiation - a follow-up study. Clinical Endocrinology 199951 281-284.

7 Greenman Y, Ouaknine G, Veshchev I, Reider-Groswasser II, Segev Y \& Stern N. Postoperative surveillance of clinically nonfunctioning pituitary macroadenomas: markers of tumour quiescence and regrowth. Clinical Endocrinology $2003 \mathbf{5 8}$ 763-769.

8 Marsden JE \& Tromba AJ. Vector Calculus, Fifth Edition. 2 New York: W.H. Freeman and Company, 2003 (ch 5, pp 317-367).

9 Ratkowsky DA. Handbook of Nonlinear Regression Models Statistics: Textbooks and Monographs. vol 107 New York: Marcel Dekker, 1990.

10 Ezzat S, Asa SL, Couldwell WT, Barr CE, Dodge WE, Vance ML \& McCutcheon IE. The prevalence of pituitary adenomas: a systematic review. Cancer 2004101 613-619.

11 Daly AF, Rixhon M, Adam C, Dempegioti A, Tichomirowa MA \& Beckers A. High prevalence of pituitary adenomas: a crosssectional study in the province of Liège, Belgium. Journal of Clinical Endocrinology and Metabolism 200691 4769-4775.
12 Dekkers OM, Hammer S, de Keizer RJW, Roelfsema F, Schutte PJ, Smit JWA, Romijn JA \& Pereira AM. The natural course of nonfunctioning pituitary macroadenomas. European Journal of Endocrinology $2007 \mathbf{1 5 6} 217-224$.

13 Sanno N, Oyama K, Tahara S, Teramoto A \& Kato Y. A survey of pituitary indicentaloma in Japan. European Journal of Endocrinology $2003149123-127$.

14 Araujo RP \& McElwain DLS. A history of the study of solid tumour growth: the contribution of mathematical modelling. Bulletin of Mathematical Biology 200466 1039-1091.

15 Deisboeck TS, Berens ME, Kansal AR, Torquato S, StemmerRachamimov AO \& Chiocca EA. Pattern of self-organization in tumour systems: complex growth dynamics in a novel brain tumour spheroid model. Cell Proliferation 200134 115-134.

16 Marusic M, Bajzer Z, Freyer JP \& Vuk-Pavlovic S. Analysis of growth of multicellular tumour spheroids by mathematical models. Cell Proliferation 199427 73-94.

17 Swanson KR, Bridge C, Murray JD \& Alvord EC. Virtual and real brain tumors: using mathematical modeling to quantify glioma growth and invasion. Journal of the Neurological Sciences 2003216 $1-10$.

18 Landry J, Freyer JP \& Sutherland RM. A model for the growth of multicellular spheroids. Cell and Tissue Kinetics 198215 585-594.

19 Steel GG \& Lamerton LF. The growth rate of human tumours. British Journal of Cancer 196620 74-86.

20 Cho KG, Hoshino T, Nagashima T, Murovic JA \& Wilson CB. Prediction of tumor doubling time in recurrent meningiomas. Journal of Neurosurgery $1986 \mathbf{6 5} 790-794$.

21 Yamashita T \& Kuwabara T. Estimation of rate of growth of malignant brain tumors by computed tomography scanning. Surgical Neurology 198320 464-470.

22 Nakamura M, Roser F, Michel J, Jacobs C \& Samii M. The natural history of incidental meningiomas. Neurosurgery 200353 62-71.

23 Ekramullah SM, Saitoh Y, Arita N, Ohnishi T \& Hayakawa T. The correlation of Ki-67 staining indices with tumour doubling times in regrowing non-functioning pituitary adenomas. Acta Neurochirurgica 1996138 1449-1455.

24 Tanaka Y, Hongo K, Tada T, Sakai K, Kakizawa Y \& Kobayashi S. Growth pattern and rate in residual nonfunctioning pituitary adenomas: correlations among tumor volume doubling time, patient age, and MIB-1 index. Journal of Neurosurgery $2003 \mathbf{9 8}$ 359-365.

25 Honegger J, Prettin C, Feuerhake F, Petrick M, Schulte-Mönting J \& Reincke M. Expression of Ki-67 antigen in nonfunctioning pituitary adenomas: correlation with growth velocity and invasiveness. Journal of Neurosurgery 200399 674-679.

Received 16 December 2007

Accepted 17 December 2007 